\title{
Extranodal Tuberculosis of Head and Neck: A Series of 29 Cases
}

\section{Sameh Mezri*, Ghassen Chebbi, Manel Chebbi, Sabrine Maamouri, Rania Ben Mhamed, Khemaies Akkari}

ENT Department, Military Hospital of Tunis, Tunis, Tunisia

Email: *mezrisameh@gmail.com

How to cite this paper: Mezri, S., Chebbi, G., Chebbi, M., Maamouri, S., Mhamed, R.B. and Akkari, K. (2019) Extranodal Tuberculosis of Head and Neck: A Series of 29 Cases. Journal of Tuberculosis Research, 7 , 148-158.

https://doi.org/10.4236/jtr.2019.73015

Received: June 26, 2019

Accepted: September 22, 2019

Published: September 25, 2019

Copyright $\odot 2019$ by author(s) and Scientific Research Publishing Inc. This work is licensed under the Creative Commons Attribution International License (CC BY 4.0).

http://creativecommons.org/licenses/by/4.0/

\begin{abstract}
Background: Despite a re-increase in the incidence of tuberculosis, extranodal location on head and neck remains rare. Clinical symptomatology is non-specific and even data from paraclinical explorations often pose the problem of differential diagnosis with tumor pathology. Methods: We report a series of 29 cases of extranodal tuberculosis collected over a period of 32 years [1986-2018] in ENT department of the military hospital of Tunis. Results: Rhinosinusopharyngeal involvement was predominant (45\%) followed by glandular involvement (14\%). Diagnosis was histopathological in most cases. Concomitant pulmonary tuberculosis was detected in two cases and spinal lumbar in one case. Treatment was medical in all cases. Conclusion: Clinical presentation of extranodal tuberculosis is various and non-specific. Neoplastic pathology is the main differential diagnosis. However, diagnosis should be systematically raised in endemic countries. Treatment is essentially medical.
\end{abstract}

\section{Keywords}

Tuberculosis, ENT, Extranodal

\section{Introduction}

Tuberculosis is a chronic specific infectious disease caused by mycobacteria: Koch bacillus $(B K)$. In Tunisia, it is still endemic and represents one of the public health problems with the increase of resistant forms. Lymph node involvement is the most common ENT location [1]. However, extranodal locations, although rare, are not exceptional. Nonspecific and polymorphic clinical presentation is often responsible for diagnostic difficulties that may conflict with tumour pathology or other chronic non-infective and infective pathological conditions. 
The aim of our study is to expose the different clinical forms of extra nodal tuberculosis in ENT area and to analyze its epidemiological, clinical and diagnosis specificities.

To our knowledge, this is the largest published series of extranodal tuberculosis of the head and neck.

\section{Patients and Methods}

We conducted a descriptive retrospective study on 29 cases of extranodal tuberculosis of the head and neck diagnosed and treated in the ENT department of the Military Hospital of Tunis collected over a period of 32 years [1986-2018].

The diagnosis, suspected because of the epidemiological and clinical context, was based on data from histopathological examination that revealed chronic granulomatous inflammatory exudates wit centrally caseating necrosis or by isolating BK on the bacteriological samples.

Patients treated on elements of presumption without diagnostic evidence and those lost to follow-up before the end of treatment were excluded from this study.

During the period before 2009, patients received anti-tuberculosis therapy based on quadritho therapy (isoniazid, rifampin, pyrazinamide and streptomycin/ethambutol) for 2 months followed by bitherapy (isoniazid, rifampin). After this date, the treatment is based on combined treatment HRZE followed by bitherapy HR. In all cases, the minimum duration of medical treatment was 8 months.

Epidemiological factors, clinical condition at presentation, biological and radiologic explorations underwent according to each location, methods of biopsy, treatment modalities, follow-up, and outcome were reported.

\section{Results}

We collected 29 cases of extranodal tuberculosis of the ENT sphere. The average age of our population was 42 years old with extremes ranging from 15 to 65 years old. There were 11 men and 18 women with a sex ratio of 0.61 .

The average time of consultation was 12 months [ 2 months to 25 months]. Four of our patients had a history of pulmonary tuberculosis and correct vaccination was reported by 20 patients. A family history of tuberculosis was reported in five cases with three pulmonary locations and two cervical locations. Only one patient was diabetic. Functional symptoms varied with the affected site (Table $1)$.

\subsection{Rhino-Sino-Pharyngeal Involvement}

It represents the most common location in our series. Nasal endoscopy noted a pseudo-tumoral budding formation of the cavum in six cases (46\%), and a granulomatous mucosa in other cases. The biopsy confirmed the diagnosis of tuberculosis in all cases. Sinus involvement was isolated in three cases (Figure 1). No extra ENT tuberculosis location was found. 


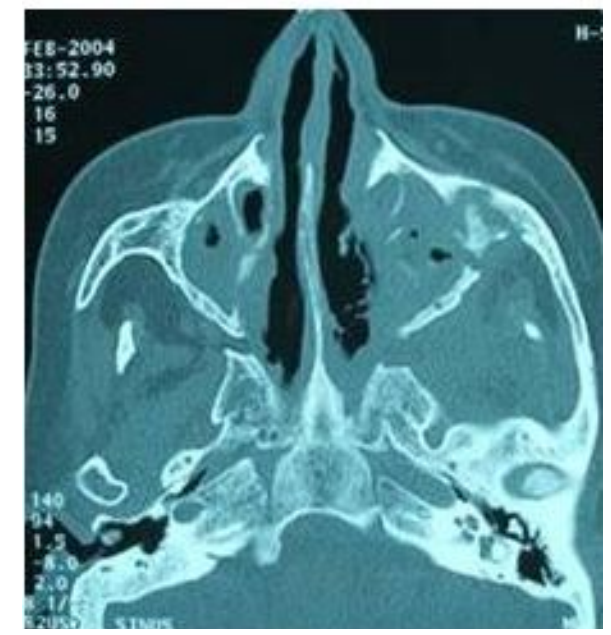

Figure 1. Facial CT scan (axial cut): filling of the two maxillary sinuses with reshaping and bone lysis of sinus and intersinuso-nasal walls.

Table 1. Functional symptoms according to the location of tuberculosis.

\begin{tabular}{|c|c|c|c|c|}
\hline \multirow[b]{2}{*}{ Locations } & \multirow[b]{2}{*}{ n (\%) } & \multicolumn{2}{|c|}{ Socio-demographic features } & \multirow[b]{2}{*}{ functional signs } \\
\hline & & $\begin{array}{c}\text { Average } \\
\text { age (years) }\end{array}$ & $\begin{array}{l}\text { Sex-ratio } \\
(M / F)\end{array}$ & \\
\hline \multicolumn{5}{|l|}{ Upper airways location } \\
\hline Rhino-sinuso-pharyngeal & $13(45)$ & 40 & $3 / 10$ & $\begin{array}{l}\text { Blood-stained (69\%), NO } \\
(54 \%) \text {, auricular fullness } \\
(46 \%), \text { purulent resistant } \\
\text { rhinorrhea }(30 \%) \text {, cervical } \\
\text { lymph nodes }(30 \%)\end{array}$ \\
\hline Tonsil & $2(7)$ & 56 & $2 / 0$ & $\begin{array}{l}\text { Odynophagia, alteration } \\
\text { of the general condition }\end{array}$ \\
\hline Larynx & $2(7)$ & 54 & $2 / 0$ & $\begin{array}{l}\text { Chronic dysphonia, } \\
\text { high dysphagia, resistant } \\
\text { productive cough }\end{array}$ \\
\hline \multicolumn{5}{|l|}{ Glandular location } \\
\hline Thyroid & $5(17)$ & 42 & $0 / 5$ & Cervical swelling \\
\hline Parotid & $5(17)$ & 51 & $3 / 2$ & Lateral face swelling \\
\hline \multicolumn{5}{|l|}{ Bone location } \\
\hline Mandible & $1(3.5)$ & 30 & $1 / 0$ & Pain of the chin \\
\hline $\begin{array}{l}\text { Cervical spine } \\
\text { (Pott's disease) }\end{array}$ & $1(3.5)$ & 64 & $0 / 1$ & Odynophagia, paraplegia. \\
\hline
\end{tabular}

n: number of cases; NO: Nasal obstruction; M: Men; F: Female.

In sinus locations, we associated endoscopic surgical treatment. Evolution was favorable in 11 cases under well-managed antituberculous treatment for 9 to 13 months. Two patients presented resistance to treatment with pulmonary involvement during treatment and treatment was adapted according to the anti-biogram of new bacteriological sampling. 


\subsection{Tonsillar Location}

Clinical examination revealed an asymmetry in palatal tonsil size that was erythemato-pultaceae associated with cervical lymphadenopathy in one case. Anatomopathological examination of tonsillectomy product concluded to granulomatous and necrotizing tonsillitis (Figure 2). No other location of tuberculosis was found. Patients had a treatment for 10 and 12 months respectively with no recurrence after 12 months follow-up.

\subsection{Laryngeal Location}

Direct laryngoscopy revealed an ulcero-budding and infiltrating lesion of the epiglottis in one case and granulomatous lesions of the laryngeal vestibule (Figure 3) with an erythematous epiglottis and edema in the second case. The rest of assessment showed concomitant active pulmonary tuberculosis in both cases. Conventional antituberculosis treatment for 12 months has been prescribed with no recurrence after 24 months follow-up.

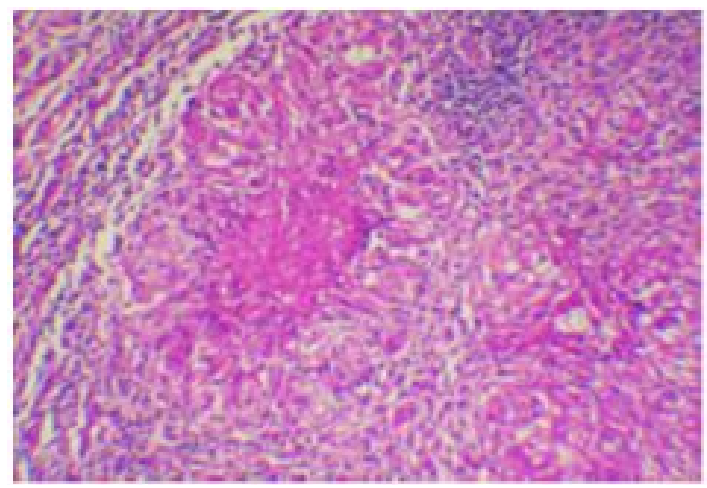

Figure 2. Pathology slide: Tonsillar tissue with huge gigantocellular granuloma and caseous necrosis.

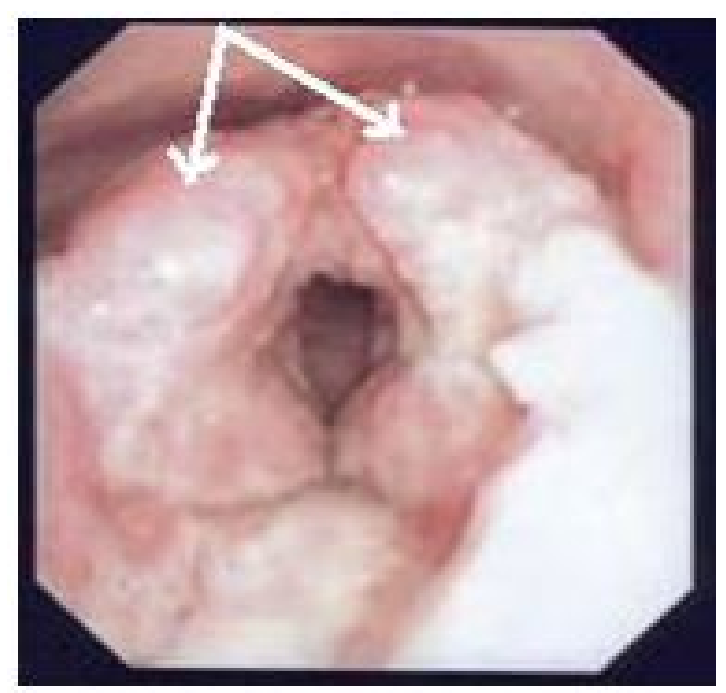

Figure 3. Endoscopic view: Glottic and supra-glottic granulomatous lesion corresponding to laryngeal tuberculosis. 


\subsection{Parotid Localization}

The clinical examination objectified a nodular renitent formation (two cases) and nodular hard consistency (three cases). Local inflammatory signs were noted in two cases. The stenon was free with clear saliva in all cases and no patient had peripheral facial palsy. Imaging (ultrasound, CT or resonance imaging) had objectified a necrotic tissue in the parotid gland in all cases. Diagnosis was suested by histopathological examination of exofacial parotidectomy in three cases and by isolating $B K$ in pus aspiration in two cases. A recovery was reached through a 12 months average antituberculous treatment.

\subsection{Thyroid Location}

Diagnosis was made on a lobectomy piece for a thyroid nodule with a banal appearance in three cases and on a total thyroidectomy piece with lymph node dissection for suspicious multi nodular goitre (EU-TIRADS 5 on ultrasound) in two cases. Papillary cancer of the thyroid was associated in one case. All patients had received conventional anti-tuberculosis treatment for 12 to 14 months with reassuring clinical control in all cases.

\subsection{Mandibular Location}

Examination was strictly normal except for sensitivity of the left mandible. X-ray showed lacunary image at the mandibular symphysis. CT scan objectified a left symphysis osteolysis with cortex rupture (Figure 4). Histopathological study of an excisional biopsy confirmed its tuberculous nature. Patient received antituberculous treatment for 12 months. After a two-year follow-up, control CT did not show signs of recurrence.

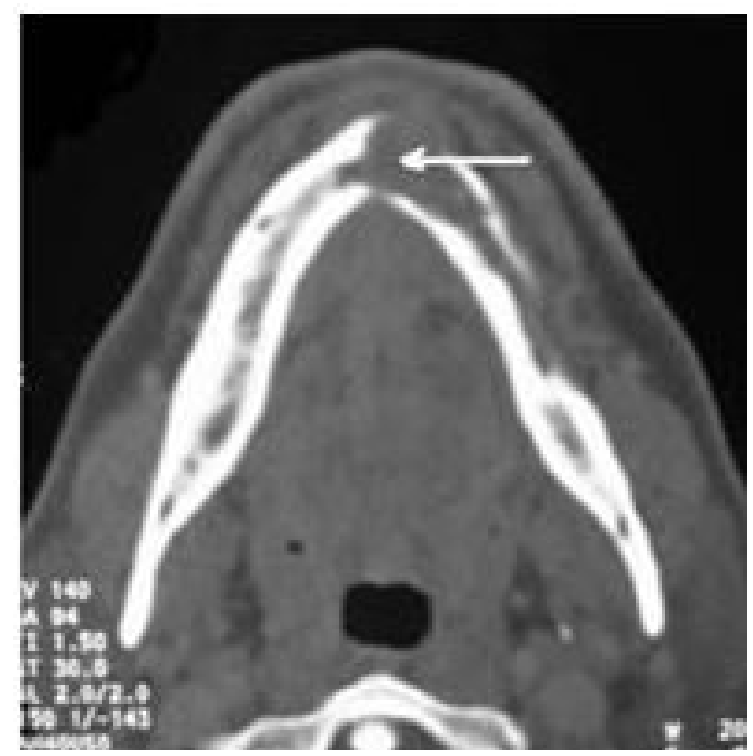

Figure 4. Mandible CT scan: An osteolytic process of the left hemi-mandible extending to the symphysis, breaking the cortex by places, with a tissue matrix density and measuring $3 \times 5 \times 1.5 \mathrm{~cm}$. 


\subsection{Cervical Pott's Disease}

In addition to a flaccid paraplegia, clinical examination revealed a right-lateral pharyngeal bulge extended from the nasopharynx to the oropharynx with normal-looking mucosa. Cervical and medullary MRI revealed a mass developed in the soft tissues in front of $\mathrm{C} 1$ and $\mathrm{C} 2$ invading the cervical cord as well as lysis of the D6 - D7 bodies (Figure 5).

We performed an abscess flattening by the oropharyngeal route and the bacteriological study isolated the BK. An anti-tuberculous treatment was prescribed for 18 months with an immobilization of the cervical spine by a neck brace. Despite the sterilization of the tuberculosis site, the patient still kept the paraplegia.

\section{Discussion}

Tuberculosis remains a major global health problem and the fight against tuberculosis is a priority for WHO. It is the second leading cause of death from infectious disease after HIV [1] [2].

HIV infection, the emergence of resistant strains, the large migratory flow of populations and the improvement of data collection, favor its current upsurge [3].

However, extranodal tuberculosis of the ENT sphere remains rare, representing less than $1 \%$ of all locations [4] [5] [6] [7]. In addition to the increased incidence, there is also a change in the clinical presentation. Indeed, the ENT locations have for a long time been secondary and/or associated with pulmonary forms, whereas currently primitive ORL forms, although rare, are not exceptional.

The contamination may be secondary to local inoculation (by inhalation of bacilliferous dust or scratching lesion) or to inoculation by blood or lymphatic system from an unknown or a recognized site [8].

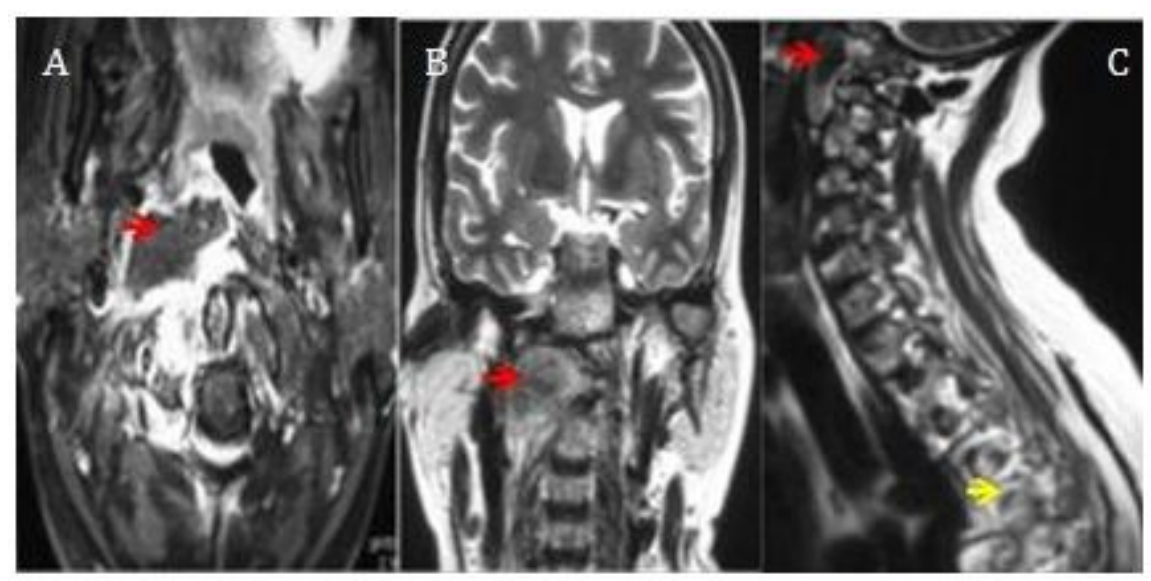

Figure 5. MRI of cervical and dorsal spine showing soft tissue mass developed ahead of C1 - C2 compressing the right lateral wall of the nasopharynx. This mass is in hypersinal on T2 weighted images with peripheral enhancement. Lysis of the vertebral body of D6 and D7. Anterior epidural lesion exerting a mass effect on spinal cord wit intense enhancement after gadolinium injection. 
The multiple mechanisms of inoculation thus explain the topographic diversity and the possibility of forms with multiple locations.

- Primary nasopharyngeal tuberculosis, defined as isolated tuberculous infection of the nasopharynx, is rare [9] accounting for $0.12 \%$ of all TB sites in Contant series and appears to be more common in young subjects [10]. It was the most frequent location in our patients found in $45 \%$ of cases. The functional symptomatology is not specific, dominated by nasal obstruction and epistaxis, less frequently otological signs such as sero-mucous otitis. Associated cervical lymphadenopathies have been reported in $50 \%$ to $70 \%$ of cases according to series [11] [12]. As seen in our study, nasal endoscopy commonly shows a budding or ulcero-budding formation of the posterior wall [13].

In a country of medium endemicity for undifferentiated carcinoma of the nasopharynx (UCNT) like ours, this aspect often evokes cavum neoplasia. Radiological images are not specific and can lead to malignant process hence the interest of biopsies.

- Primary nasosinusal tuberculosis was first described by Morgani in 1761 on autopsy data [14] but it is still rare with a frequency of $1 \%$ of tuberculous locations of the head and neck [14]. The proposed route of contamination is a secondary contamination by inhalation of the tubercle bacillus from a neighboring site, especially pulmonary. Primary involvement is very rare. In our series, three sinus lesions were highlighted and were all associated with a nasopharyngeal location.

In nasal fossae, lesion mainly concerns the inferior turbinate and the cartilaginous portion of the septum [15].

The lesions may take the form of granulomatous proliferative tissue, polypoid or ulceration that may be confused with other granulomatoses whether infectious or tumoral. Prognosis depends on the site of lesions (more reserved in case of bone involvement) and the early management of the case. All of our patients with sinus involvement received endoscopic surgical treatment followed by standard anti-tuberculosis treatment.

- In its oropharyngeal location, tonsil involvment remains rare, representing $16 \%$ of cases in the Gassab series [16]. The common symptomatology is reminiscent of angina. Clinical examination may indicate chronic painful ulceration or pseudotumor hypertrophy suggesting a cancer or lymphoma [17] [18]. The association with cervical lymph node tuberculosis is classic, reported in up to $90 \%$ of cases, explained by the lymphoid richness of the pharynx (Waldeyer's ring). Isolated pharyngeal involvement remains exceptional, and it is necessary to search for an associated location such as involvement of the cavum, larynx and/or evolutionary pulmonary which is reported in $30 \%$ to $96 \%$ according to the series [19]. For us two patients, no other involvement was associated.

- Laryngeal tuberculosis is relatively rare, representing almost $1 \%$ of the extra-pulmonary locations of the disease [20]. It is more common in young 
adults between the ages of 20 and 50 years, diabetics and people with AIDS [21] [22].

Endoscopic aspects can take many forms and are confusing in budding ulcer and infiltrating ulcer forms with malignant pathology. However, the coexistence of both tuberculosis and cancerous lesions is not uncommon [22]. The lesions predominate in the glottis, for our patients the lesion was supra-glottal.

In all cases, an endoscopy examination with an anatomopathological and bacteriological study is necessary to identify the BK, make the diagnosis of laryngeal tuberculosis and eliminate neoplastic pathology or to confirm the association. The chest $\mathrm{x}$-ray must be done systematically, it is pathological in $50 \%$ to $100 \%$ of cases [23], it was pathological in our two patients. Antitubercular chemotherapy is sufficient to ensure healing. Surgery is limited to airway unblocking by laser vaporization or micro-instruments.

- Glandular involvement with tuberculosis is rare. Parotid location is, however, the most common. Since the first case described by Von Stubenrach in 1894, just over 220 cases have been reported [24]. The infection affects parotidean parenchyma as well as periglandular lymph nodes and spreads haematogenously or lymphatically. Patients usually have a nodular or diffuse parotid swelling mostly unilateral, which may be accompanied by pain and trismus, but the discovery is often a histological surprise on a piece of excision. The involvement of the facial and trigeminal nerve has been reported in the literature. The presence of cervical lymphadenopathy is common. Salivary secretion is not infected by koch bacillus.

Diagnostic confirmation involves parotid puncture, as in two of our cases, or excisional biopsy by parotidectomy [25].

Thyroid location is very rare even in endemic countries representing $0.1 \%$ to $0.4 \%$ of all locations of the disease [26]. This rarity is explained by the good oxygenation of the thyroid parenchyma and the tuberculostatic role of the thyroid hormones. In our study, diagnosis was unexpected in all cases. The treatment is mainly medical. In case of collection, drainage is sufficient, thyroidectomy remains exceptional. However, the association with thyroid cancer is not exceptional [26].

- Mandibular tuberculosis is rare, representing less than $2 \%$ of skeletal tuberculosis sites. The clinical aspects are varied and misleading, source of diagnostic delay. Local signs become evident only at advanced stages of fistulized or non-fistulated abscess [27]. Panoramic dental X-ray often shows an extensive osteolytic lesion, or combining lysis and condensation with usually fuzzy limits. The CT aspects are not specific: peri-odontitis with bone loss, bone remodeling with osteolysis and osteo condensation, and pre-mandibular soft tissue abscess [28]. The diagnosis can only be histological.

- Although common in endemic and disadvantaged countries, tuberculosis location in the upper cervical spine is very rare estimated at $1 \%$ of spinal locations [29]. Clinical presentation is non-specific ranging from spasmodic torticollis to tetraplegia. Diagnosis is often made at the stage of neurological 
complications as in our patient. On CT scan, the typical aspect associates $\mathrm{C} 1$ $\mathrm{C} 2$ vertebral destruction with soft tissue swelling. MRI allows a better analysis of the extent of lesions, the state of the occipito-cervical hinge and the location of the odontoid [30]. Diagnosis is mainly histo-bacteriological but can be retained on elements of presumption. The treatment is medical based on antituberculous drugs and immobilization of the cervical spine. Surgery is indicated if important bone destruction with spinal instability. Our patient remained paraplegic after 24 months follow-up.

\section{Conclusion}

Although the worldwide incidence of tuberculosis disease is increasing, extranodal locations of the ENT sphere remain rare. Clinically they pose diagnostic difficulties with neoplastic pathology. Treatment is mainly medical based on anti-tuberculosis drugs. Surgery has a limited place in pseudo-tumor forms or in cases of complications with favorable evolution in most cases.

\section{Conflicts of Interest}

The authors declare no conflicts of interest regarding the publication of this paper.

\section{Authors' Contributions}

All authors' contributed to elaborate the manuscript and have all approved final version of manuscript.

\section{References}

[1] Chouaid, C. (2006) Actualités de la tuberculose. Revue des Maladies Respiratoires, 23, 80-85. https://doi.org/10.1016/S0761-8425(06)72006-X

[2] World Health Organization (2008) Global Tuberculosis Control: Surveillance, Planning, Financing.

[3] World Health Organization (2016) Global Tuberculosis Report 2016. 1. Tuberculosis Epidemiology. 2. Tuberculosis, Pulmonary Prevention and Control. 3. Tuberculosis Economics. 4. Tuberculosis, Multidrug-Resistant. 5. Annual Reports.

[4] Wookyng, M., Moon Hee, H., Chang, J., et al. (1997) CT and MR Imaging of Head and Neck. Tuberculosis Radiographies, 17, 391-402.

https://doi.org/10.1148/radiographics.17.2.9084080

[5] Mahfoudhi, M., Khamassi, K., Turki, S. and Kheder, A. (2015) Difficulté diagnostique d'une tuberculose laryngée isolée chez une femme diabétique. Pan African Medical Journal, 21, 106. https://doi.org/10.11604/pamj.2015.21.106.6361

[6] Bouchentouf, R., Bouaity, B., Touati, M., Benjelloun, A. and Aitbenasser, M.A. (2013) La tuberculose nasosinusienne primaire: A propos d'un cas. Pan African Medical Journal, 14, 29. https://doi.org/10.11604/pamj.2013.14.29.1039

[7] Kharoubi, S. (1998) La tuberculose pharyngée: Etude analytique, à propos de 10 cas. Revue de Laryngologie-Otologie-Rhinologie, 3, 203-207.

[8] Clément, P., Le Guyadec, T., Zalagh, M., Gauthier, J., N’Zouba, L. and Kossowski, M. (2005) Manifestations bucco-naso-sinusiennes des maladies infectieuses spécifiques. EMC-Oto-rhino-laryngologie, 2, 198-217. 
https://doi.org/10.1016/j.emcorl.2004.11.002

[9] Contant, A., Fantan, Y., Peraldi, R. and Acquaviva, F. (1995) Tuberculose Nasale: A propos d'un cas. Revue de Laryngologie-Otologie-Rhinologie, 116, 225-228.

[10] Chopra, R.K., Kerner, M.M. and Caleaterra, T.C. (1994) Primary Nasopharyngeal Tuberculosis: A Case Report and Review of This Rare Entity. Otolaryngology-Head and Neck Surgery, 111, 820-823. https://doi.org/10.1177/019459989411100620

[11] Waldron, J., Van Hasselt, C.A., Skinner, D.W. and Arnold, M. (1992) Tuberculosis of the Nasopharynx: Clinicopthological Features. Clinical Otolaryngology and Allied Sciences, 17, 57-59. https://doi.org/10.1111/j.1365-2273.1992.tb00989.x

[12] Akkari, K., Sethom, A., Ouni, H., Brahem, H., et al. (2005) Tuberculose ORL Extra Ganglionnaire. Journal Tunisien d ORL et de Chirurgie Cervico-Faciale, 15, 21-26.

[13] Ellouze, R., Tabbane, F. and Benattia (1973) Aspects pseudo-tumoraux de la tuberculose extra-pulmonaire en dehors des ganglions. La Tunisie Médicale, 51, 147-150.

[14] Slutsker, L., Castro, K.G., Ward, J.W., et al. (1993) Epidemiology of Extrapulmonary Tuberculosis among Persons with Aids in the United States. Clinical Infectious Diseases, 16, 513-518. https://doi.org/10.1093/clind/16.4.513

[15] Ravi, N., Juma Al, K. and Kanchanmala, G. (2004) Primary Nasal Tuberculosis: A Case Report. Ear, Nose \& Throat Journal, 44, 23-34.

[16] Gassab, E., Kedous, S., Berkaoui, E., Sayeh, N., Harrathi, K., Koubaa, J., et al. (2010) Tuberculose extra ganglionnaire de la tête et du cou. Journal Tunisien d ORL et de Chirurgie Cervico-Faciale, 24, 26-30. https://doi.org/10.4314/jtdorl.v24i1.64197

[17] Moulonguet, L., Delguidice, P. and Chauvin, J.L. (1995) L'angine tuberculeuse: A propos d'un cas au Sénégal. The Annals of Otology, Rhinology, and Laryngology, $112,78-79$.

[18] Awad, M., Al-Serhani, A.M. and Al-Mazrou, K. (2000) Pharyngeal Tuberculosis. American Journal of Otolaryngology, 22, 236-240. https://doi.org/10.1053/ajot.2001.24818

[19] Kharoubi, S. (2015) Tuberculose de l'amygdale palatine: A propos d'un cas. Research fr, 2, 1402.

[20] Oudidi, A., Ridal, M., Cherkaoui, A. and El Alami, M.N. (2005) Tuberculose laryngée. À propos de neuf cas. La Lettre d Oto-rhino-laryngologie et de chirurgie cervico-faciale, 296, 18-20.

[21] Yang, Z., Kong, Y., Wilson, F., et al. (2004) Identification of Risk Factors for Extrapulmonary Tuberculosis. Clinical Infectious Diseases, 38, 199-205. https://doi.org/10.1086/380644

[22] El Ayoubi, F., Chariba, I., El Ayoubi, A., Chariba, S. and Essakalli, L. (2014) Primary Tuberculosis of the Larynx. European Annals of Otorhinolaryngology, Head and Neck Diseases, 131, 361-364. https://doi.org/10.1016/j.anorl.2013.10.005

[23] Chen-Chi, W., Chang-Chun, L., Ching-Ping, W., Shih-An, L. and Rong-San, J. (2007) Laryngeal Tuberculosis: A Review of 26 Cases. Otolaryngology-Head and Neck Surgery, 137, 582-588. https://doi.org/10.1016/j.otohns.2007.04.002

[24] Chouai, M., Lachkar, A., Abdenbi Tsen, A., El Ayoubi, F. and Ghailan, R.M. (2018) Tuberculose parotidienne: Un diagnostic oublie, à propos de 2 cas et revue de littérature. Pan African Medical Journal, 29, 203. https://doi.org/10.11604/pamj.2018.29.203.14682

[25] Benhammou, A., El Ayoubi, A., Benbouzid, M.-A., Boulaich, M., Essakali, L. and Kzadri, M. (2007) Tuberculose primaire de la glande parotide. Archives de 
Pédiatrie, 14, 1206-1209. https://doi.org/10.1016/j.arcped.2007.06.012

[26] Andriarimanana hery nirina, R., Andy, M., Rakooto, F.A., Pierre Georges, R., Tiana, R. and Fred, R. (2007) La tuberculose de la glande thyroïdienne: A propos de deux cas. Journal ORL de la société française d' ORL et CCF, 92, 274-320.

[27] N'Golet, A., N'Golet Ocini, L., Mboussa, J. and N'Gouoni, B.G. (2007) Primary Mandibular Tuberculosis. Revue de Stomatologie et de Chirurgie Maxillo-Faciale, 108, 65-67. https://doi.org/10.1016/j.stomax.2005.11.002

[28] Boussel, L., Marchand, B., Blineau, N., et al. (2002) Imaging of Osteoarticular Tuberculosis. Journal of Radiology, 83, 1024-1034.

[29] Jain, A.K., Kumar, S. and Tuli, S.M. (1999) Tuberculosis of Spine (C1 to D4). Spinal Cord, 37, 362-369. https://doi.org/10.1038/sj.sc.3100833

[30] Bouabdellah, M., Bouzidi, M., Kamoun, S., Mohamed, F., Amara, K., Chabouni, L., et al. (2010) Pott's Disease of the Upper Cervical Spine (Three Cases and Literature Review). La Tunisie Medicale, 88, 847-850. 